\title{
Difference Analysis on Ecological Civilization Construction in Reclamation Area
}

\author{
PAN Shasha, ZHANG Chuanhui* \\ College of Economics and Management, Tarim University; Alar City of Xinjiang, China \\ *.Department of Ideological and Political Education, Tarim University; Alar City of Xinjiang, China \\ chuanhui@163.com
}

Keywords: Reclamation Area; Ecological Civilization Construction; Difference Analysis.

\begin{abstract}
In order to scientifically evaluate the ecological civilization construction situations in reclamation area, this paper builds a frame system covering three links of pressure - status response and containing 21 indexes on the basis of PSR model. Meanwhile, an empirical analysis is made for the ecological civilization construction situations and coordination degree in 14 divisions of Xinjiang Corps in 2013. The evaluation results present the following conclusions. (1) Ecological civilization construction presents regional difference in Xinjiang Corps and ecological civilization construction is a progressive course from north Xinjiang to south Xinjiang. The coordination degree of ecological civilization construction in north Xinjiang is higher than that in south Xinjiang, which almost reflects the regional situations of ecological civilization construction in Xinjiang Corps. According to various subsystems, the ecological system pressure in south Xinjiang is smaller than that in north Xinjiang, the health status of ecological system in north Xinjiang is better than that in south Xinjiang, and the management level of ecological environment in north Xinjiang is higher than that in south Xinjiang. (2) North Xinjiang is the population and economic center of Xinjiang Corps and it bears the main area of social \& economic construction and resident gathering. Therefore, a great effort should be made to realize a healthy ecological system and enhance resource and energy utilization efficiency in north Xinjiang. At the same time, the ecological environment must be protected and ecological civilization system construction should be strengthened. South Xinjiang should aim to protect the vulnerable ecological environment, preserve its original ecological environment, and improve its resource and energy utilization efficiency and environmental pollution control ability.
\end{abstract}

\section{Introduction}

Scientific constitution for an index system of evaluating ecological civilization construction is an important method to weigh and appraise ecological civilization construction[1]. Development of ecological civilization was mentioned at the 17th CPC National Congress for the first time, and ecological civilization construction was brought into "five-in-one" layout at the 18th CPC National Congress. This has fully reflected the aspiration and determination of CPC to enhance ecological civilization construction and socialist modernization.

Under this general background, domestic scholars have made many referable achievements in studies on ecological civilization construction, especially studies on evaluation index system. Domestic scholars have proposed multiple ecological civilization evaluation index systems covering province, region and city, and carried out a series of empirical researches. GUAN Yanzhu, ZHENG Jianhua and ZHUANG Shijian[2] (2007), DU Yu and LIU Junchang[3] (2009), et al. established an interactive relationship between man and nature and made an empirical analysis for the accessibility of ecological civilization construction indexes. YAN Yezhou and CHENG Jinhua [4] (2013), DIAO Shangdong and LIU Yunzhong[5] (2013), et al. analyzed the ecological civilization construction index system in Guangzhou City via index model. Besides, ZHANG Chuanhui, ZHAO 1 
Jinling, HAN Wei[6-8] (2007, 2008, 2014), et al. discussed some ideas about ecological civilization from Marxist natural view, Chinese traditional natural view, and Xi Jinping's thought. The common research methods include entropy evaluation method, principal component analysis method, and comprehensive evaluation method. Western countries entered the industrialization period at an early time, so most overseas scholars mainly make studies from the aspect of environmental sustainability. Their studies can provide a good reference for this paper.

As a special region, Xinjiang Corps possesses a special geographical position and bears multiple functions. As a result, Xinjiang Corps needs a sustainable plan in transformation of agricultural development mode, adjustment of industrial structure, and sustainable development and utilization of resources, so as to effectively promote ecological civilization construction. Therefore, scientific evaluation of ecological civilization construction is beneficial to comprehensive construction of a well-off society in Xinjiang Corps. Moreover, it can point out the direction of modern ecological construction for agriculture more clearly, and realize good and sustainable development of social economy and environment[11].

\section{Construction of ecological civilization evaluation index system under the perspective of ecological system}

PSR model. PSR (pressure - status - response) model is one of the models that can evaluate resource utilization and sustainable development. This model is based on causal relationship, and the three links of pressure - status - response will influence and restrict each other; it reflects the whole process of decision-making and countermeasure development. According to "pressure status - response" model frame, we construct a regional ecological civilization construction evaluation index system composed of three subsystems (ecological system pressure, health status and ecological environment management level) including 21 indexes under the perspective of ecological system as per the comprehensive, typical and quantitative operation principle on the basis of PSR frame model (see Table 1). 
Table 1 PSR model under the framework of evaluation index system of Xinjiang production and construction of ecological civilization

\begin{tabular}{|c|c|c|c|c|}
\hline Element layer & Index layer & Statistical pattern & $\begin{array}{c}\text { Index } \\
\text { attribute }\end{array}$ & Index explanation \\
\hline \multirow{6}{*}{$\begin{array}{l}\text { Ecological } \\
\text { system } \\
\text { pressure X1 } \\
\text { (Pressure) }\end{array}$} & $\begin{array}{l}\text { Natural population growth rate (\%) } \\
\text { X1 }\end{array}$ & $\begin{array}{l}\text { Added value of population / gross } \\
\text { population of last year }\end{array}$ & $\begin{array}{l}\text { Constraint } \\
\text { index }\end{array}$ & $\begin{array}{l}\text { Population } \\
\text { pressure }\end{array}$ \\
\hline & $\begin{array}{l}\text { GDP per capita (Yuan) } \\
\mathrm{X} 2\end{array}$ & Statistical index & $\begin{array}{l}\text { Incentive } \\
\text { index }\end{array}$ & $\begin{array}{l}\text { Economic } \\
\text { pressure }\end{array}$ \\
\hline & $\begin{array}{l}\text { Comprehensive energy consumption } \\
\text { (ten thousand tons, standard coal) } \\
\text { X3 }\end{array}$ & Statistical index & $\begin{array}{l}\text { Constraint } \\
\text { index }\end{array}$ & $\begin{array}{l}\text { Resource and } \\
\text { environment } \\
\text { pressure }\end{array}$ \\
\hline & $\begin{array}{l}\text { Pesticide usage amount } \\
\text { X4 }\end{array}$ & Statistical index & $\begin{array}{l}\text { Constraint } \\
\text { index }\end{array}$ & $\begin{array}{c}\text { Land bearing } \\
\text { capacity pressure }\end{array}$ \\
\hline & $\begin{array}{l}\text { Agriculture plastic film usage amount } \\
\text { X5 }\end{array}$ & Statistical index & $\begin{array}{l}\text { Constraint } \\
\text { index }\end{array}$ & \\
\hline & $\begin{array}{l}\text { Agricultural fertilizer consumption } \\
\text { (pure amount) } \quad \mathrm{X} 6\end{array}$ & Statistical index & $\begin{array}{l}\text { Constraint } \\
\text { index }\end{array}$ & \\
\hline \multirow{9}{*}{$\begin{array}{l}\text { Ecological } \\
\text { system health } \\
\text { status X2 } \\
\text { (status) }\end{array}$} & $\begin{array}{l}\text { Energy consumption of production } \\
\text { valve (ton, standard coal) } \quad \mathrm{X} 7\end{array}$ & $\begin{array}{l}\text { Total energy consumption of enterprises / } \\
\text { total industrial output value of enterprises }\end{array}$ & $\begin{array}{l}\text { Constraint } \\
\text { index }\end{array}$ & \\
\hline & $\begin{array}{l}\text { Industrial added value rate (\%) } \\
\text { X8 }\end{array}$ & $\begin{array}{l}\text { Industrial added value (current price) / } \\
\text { total industrial output (current price) } \times \\
100 \%\end{array}$ & $\begin{array}{l}\text { Incentive } \\
\text { index }\end{array}$ & \\
\hline & Net income of farmer (Yuan) $\quad$ X9 & Statistical index & $\begin{array}{l}\text { Incentive } \\
\text { index }\end{array}$ & \\
\hline & $\begin{array}{l}\text { Total industrial output value (100 } \\
\text { million Yuan) X10 }\end{array}$ & Statistical index & $\begin{array}{l}\text { Incentive } \\
\text { index }\end{array}$ & \\
\hline & $\begin{array}{l}\text { Afforestation fee of state-owned and } \\
\text { state-holding enterprises }\end{array}$ & Statistical index & $\begin{array}{c}\text { Incentive } \\
\text { index }\end{array}$ & \multirow[t]{4}{*}{$\begin{array}{l}\text { Ecosystem } \\
\text { conservation } \\
\text { situation }\end{array}$} \\
\hline & $\begin{array}{l}\text { Water-saving irrigation machinery (set) } \\
\text { X12 }\end{array}$ & Statistical index & $\begin{array}{l}\text { Incentive } \\
\text { index }\end{array}$ & \\
\hline & $\begin{array}{l}\text { Wood felling quantity (hectare) } \\
\text { X13 }\end{array}$ & Statistical index & $\begin{array}{l}\text { Constraint } \\
\text { index }\end{array}$ & \\
\hline & Reservoir capacity $\left(10,000 \mathrm{~m}^{3}\right) \quad \mathrm{X} 14$ & Statistical index & $\begin{array}{l}\text { Incentive } \\
\text { index }\end{array}$ & \\
\hline & $\begin{array}{ll}\text { Death rate (\%) } & \text { X15 }\end{array}$ & Statistical index & $\begin{array}{l}\text { Constraint } \\
\text { index }\end{array}$ & $\begin{array}{l}\text { Health status of } \\
\text { residents }\end{array}$ \\
\hline \multirow{6}{*}{$\begin{array}{l}\text { Ecological } \\
\text { environment } \\
\text { management } \\
\text { level X3 } \\
\text { (response) }\end{array}$} & $\begin{array}{l}\text { Proportion of R\&D expenditure in GDP } \\
\text { (\%) X16 }\end{array}$ & Statistical index & $\begin{array}{l}\text { Incentive } \\
\text { index }\end{array}$ & $\begin{array}{c}\text { Economic } \\
\text { strengthening }\end{array}$ \\
\hline & $\begin{array}{l}\text { Recycling rate of industrial water (\%) } \\
\text { X17 }\end{array}$ & Statistical index & $\begin{array}{l}\text { Incentive } \\
\text { index }\end{array}$ & \multirow[t]{3}{*}{$\begin{array}{c}\text { Pollution control } \\
\text { ability }\end{array}$} \\
\hline & $\begin{array}{l}\text { High-tech water-saving irrigation area } \\
(10,000 \mathrm{Mu}) \quad \mathrm{X} 18\end{array}$ & Statistical index & $\begin{array}{c}\text { Incentive } \\
\text { index }\end{array}$ & \\
\hline & $\begin{array}{lc}\text { Afforestation area on barren mountains } \\
\text { and wastelands (hectare) } & \text { X19 }\end{array}$ & Statistical index & $\begin{array}{l}\text { Incentive } \\
\text { index }\end{array}$ & \\
\hline & $\begin{array}{l}\text { Profit rate of industrial costs and } \\
\text { expenses (\%) X20 }\end{array}$ & Statistical index & $\begin{array}{l}\text { Incentive } \\
\text { index }\end{array}$ & \\
\hline & \begin{tabular}{ll}
\multicolumn{3}{l}{ Broadcasting program comprehensive } \\
population coverage rate $(\%)$ & X21
\end{tabular} & Statistical index & $\begin{array}{l}\text { Incentive } \\
\text { index }\end{array}$ & \\
\hline
\end{tabular}




\section{Empirical evaluation results}

Data source and index statistics. this paper, 13 sample divisions (except the 11th division, Construction Industry Division) of Xinjiang Corps are selected as evaluation samples of this study. Data of this paper all originate from Statistical Yearbook 2014 of Xinjiang Corps, Environmental Statistical Yearbook 2014 of Xinjiang Corps, Statistical Bulletin 2013 of Xinjiang Corps, and official data issued by various divisions. In this paper, correlation analysis is conducted at first, and 11 indexes with relatively great correlation coefficients are selected from the 21 indexes and introduced into PSR model for analysis.

Weight determination. setting 13 divisions of Xinjiang Corps as samples, standardization treatment is conducted for the index data, and dimension reducing processing and empowerment are carried out via principal component analysis method. Thus comprehensive weights of various indexes are obtained; after normalization, the normalized weights are gained (Table 3).

Table 3 Principal components load matrix and weight

\begin{tabular}{|c|c|c|c|c|}
\hline Component & $\begin{array}{l}\text { First principal } \\
\text { component }\end{array}$ & $\begin{array}{c}\text { Second } \\
\text { principal } \\
\text { component }\end{array}$ & $\begin{array}{l}\text { Third principal } \\
\text { component }\end{array}$ & $\begin{array}{l}\text { Weight after } \\
\text { normalization }\end{array}$ \\
\hline Comprehensive energy consumption & 0.895 & 0.356 & -0.145 & 0.1123 \\
\hline Agriculture plastic film usage amount & 0.528 & 0.837 & 0.044 & 0.1041 \\
\hline Agricultural fertilizer consumption (pure amount) & 0.194 & 0.883 & 0.212 & 0.0762 \\
\hline $\begin{array}{l}\text { Afforestation fee of state-owned and state-holding } \\
\text { enterprises }\end{array}$ & 0.855 & 0.382 & 0.073 & 0.1184 \\
\hline Profit rate of industrial costs and expenses & -0.232 & -0.202 & 0.87 & 0.0004 \\
\hline Water-saving irrigation machinery & 0.281 & 0.291 & 0.764 & 0.0787 \\
\hline Total industrial output value & 0.859 & 0.375 & -0.02 & 0.1145 \\
\hline Proportion of RD expenditure in GDP & 0.899 & 0.313 & 0.039 & 0.1183 \\
\hline Industrial water & 0.944 & 0.206 & 0.048 & 0.1182 \\
\hline $\begin{array}{l}\text { Afforestation area on barren mountains and } \\
\text { wastelands }\end{array}$ & 0.263 & 0.568 & -0.167 & 0.0516 \\
\hline High-tech water-saving irrigation area & 0.623 & 0.753 & -0.026 & 0.1074 \\
\hline
\end{tabular}

Analysis on comprehensive scores and evaluation results. e evaluation values of index layer, system layer and objective layer are calculated according to weight coefficients and standardized evaluation values of indexes at various index layers. Meanwhile, the results are converted into centesimal system, and scores of ecological civilization construction in Xinjiang Corps are gained, as shown in Table 4. 
Table 4 Evaluation results

\begin{tabular}{|c|c|c|c|c|c|c|c|c|c|c|c|c|}
\hline \multirow{2}{*}{$\begin{array}{l}\text { Divisio } \\
\text { ns }\end{array}$} & \multicolumn{2}{|c|}{ Pressure } & \multicolumn{3}{|c|}{ Status } & \multicolumn{2}{|c|}{ Response } & \multicolumn{3}{|c|}{ Comprehensive evaluation } & \multicolumn{2}{|l|}{$\begin{array}{l}\text { Coordinati } \\
\text { on degree }\end{array}$} \\
\hline & $\begin{array}{l}\text { Evaluati } \\
\text { on value }\end{array}$ & $\begin{array}{l}\text { Centesim } \\
\text { al system }\end{array}$ & $\begin{array}{l}\text { Evaluati } \\
\text { on value }\end{array}$ & $\begin{array}{l}\text { Centesim } \\
\text { al system }\end{array}$ & $\begin{array}{l}\text { Ran } \\
\mathrm{k}\end{array}$ & $\begin{array}{l}\text { Evaluati } \\
\text { on value }\end{array}$ & $\begin{array}{l}\text { Centesim } \\
\text { al system }\end{array}$ & $\begin{array}{l}\text { Evaluati } \\
\text { on value }\end{array}$ & $\begin{array}{l}\text { Centesim } \\
\text { al system }\end{array}$ & $\begin{array}{l}\text { Ran } \\
\mathrm{k}\end{array}$ & $\begin{array}{l}\text { Evaluation } \\
\text { value }\end{array}$ & $\begin{array}{l}\text { Ran } \\
\mathrm{k}\end{array}$ \\
\hline The $9^{\text {th }}$ & -0.2247 & 56.43 & -0.0749 & 53.35 & & -0.1337 & 43.62 & -0.4333 & 52.87 & & 1.0564 & \\
\hline division & & & & & 8 & & & & & 13 & & 1 \\
\hline $\begin{array}{l}\text { The } 2^{\text {nd }} \\
\text { division }\end{array}$ & -0.1401 & 57.78 & 0.0049 & 60.43 & 6 & 0.1225 & 75.01 & -0.0127 & 59.79 & 5 & 0.9656 & 2 \\
\hline $\begin{array}{l}\text { The } 7^{\text {th }} \\
\text { division }\end{array}$ & -0.2552 & 55.95 & 0.0759 & 66.74 & 4 & -0.1085 & 46.70 & -0.2879 & 55.26 & 8 & 0.3533 & 3 \\
\hline $\begin{array}{l}\text { The } 10^{\text {th }} \\
\text { division }\end{array}$ & -0.0834 & 58.68 & -0.0805 & 52.85 & 10 & 0.1928 & 83.62 & 0.0289 & 60.48 & 4 & 0.1284 & 4 \\
\hline $\begin{array}{l}\text { The } 3^{\text {rd }} \\
\text { division }\end{array}$ & -0.5472 & 51.32 & 0.1532 & 73.61 & 2 & 0.0051 & 60.62 & -0.3889 & 53.60 & 12 & -0.0682 & 5 \\
\hline $\begin{array}{l}\text { The } 1^{\text {st }} \\
\text { division }\end{array}$ & -0.6336 & 49.95 & 0.2926 & 85.99 & 1 & 0.0321 & 63.93 & -0.3089 & 54.92 & 9 & -0.4421 & 6 \\
\hline $\begin{array}{l}\text { The } 8^{\text {th }} \\
\text { division }\end{array}$ & 1.8874 & 89.95 & 0.0966 & 68.58 & 3 & 0.0124 & 61.52 & 1.9964 & 92.87 & 1 & -0.6844 & 7 \\
\hline $\begin{array}{l}\text { The } 5^{\text {th }} \\
\text { division }\end{array}$ & -0.2287 & 56.37 & -0.0182 & 58.39 & 7 & -0.0049 & 59.40 & -0.2518 & 55.85 & 7 & -1.0012 & 8 \\
\hline $\begin{array}{l}\text { The } 6^{\text {th }} \\
\text { division }\end{array}$ & 0.4727 & 67.50 & 0.0749 & 66.65 & 5 & -0.0792 & 50.30 & 0.4684 & 67.71 & 2 & -1.0973 & 9 \\
\hline $\begin{array}{l}\text { The } 13^{\text {th }} \\
\text { division }\end{array}$ & -0.0326 & 59.48 & -0.1446 & 47.16 & 12 & 0.0053 & 60.65 & -0.1719 & 57.17 & 6 & -1.1592 & 10 \\
\hline $\begin{array}{l}\text { The } 14^{\text {th }} \\
\text { division }\end{array}$ & -0.1980 & 56.86 & -0.1577 & 45.99 & 13 & -0.0020 & 59.76 & -0.3577 & 54.11 & 11 & -1.4130 & 11 \\
\hline $\begin{array}{l}\text { The } 4^{\text {th }} \\
\text { division }\end{array}$ & 0.0907 & 61.44 & -0.0804 & 52.86 & 9 & 0.0345 & 64.22 & 0.0448 & 60.74 & 3 & -1.5930 & 12 \\
\hline $\begin{array}{l}\text { The } 12^{\text {th }} \\
\text { division }\end{array}$ & -0.1071 & 58.30 & -0.1419 & 47.39 & 11 & -0.0763 & 50.66 & -0.3253 & 54.64 & 10 & -1.6814 & 13 \\
\hline
\end{tabular}

\section{Conclusions and suggestions}

Conclusions. Firstly, regional difference is presented in pressure, status and response of ecological civilization construction in Xinjiang Corps. Ecological civilization construction in Xinjiang Corps is a progressive course from north Xinjiang to south Xinjiang. The coordination degree of ecological civilization construction in north Xinjiang is higher than that in south Xinjiang, which almost reflects the regional situations of ecological civilization construction in Xinjiang Corps. According to various subsystems, the ecological system pressure in south Xinjiang is smaller than that in north Xinjiang, the health status of ecological system in north Xinjiang is better than that in south Xinjiang, and the management level of ecological environment in north Xinjiang is higher than that in south Xinjiang. These conclusions are almost consistent with the practical situations.

Secondly, North Xinjiang is the population and economic center of Xinjiang Corps and it bears the main area of social \& economic construction and resident gathering. Therefore, a great effort should be made to realize a healthy ecological system and enhance resource and energy utilization efficiency in north Xinjiang. At the same time, the ecological environment must be protected and ecological civilization system construction should be strengthened. South Xinjiang should aim to protect the vulnerable ecological environment, preserve its original ecological environment, and improve its resource and energy utilization efficiency and environmental pollution control ability.

Suggestions. Firstly, various divisions should set up target values according to their practical conditions and build assessment criteria that meet their practical situations, so as to reduce energy 
consumption of total output value, increase resource utilization efficiency and resource output efficiency, and take the "low-consumption, efficient and intensive" road of new modern agriculture.

Secondly, divisions with healthy ecological systems should maintain their ecological dominance and develop clean energy economy at a proper time. Meanwhile, "three-high and one-low" industry should be prevented in ecologically vulnerable areas, and common people's ecological protection awareness must be enhanced. Thirdly, in regions with weak management and control ability, the pollutant emission reduction force should be increased, outdated capacity must be eliminated, repair for agricultural non-point source pollution and high consumption of water resource should be strengthened, and supervision strength of the government must be intensified.

In conclusion, ecological civilization construction in various divisions of Xinjiang Corps should not only pay attention to social and economic development pressure, land bearing capacity pressure, and resource consumption pressure, but also comprehensively consider ecological health, high-quality economic development, pollution regulation, and environmental protection ability.

\section{Acknowledgment}

Fund Program: Corps Social and scientific Fund Project (14TW08).

\section{References}

[1] LI Ganjie. Further Promotion of Ecological Civilization Construction and Improvement of Ecological Civilization Level. Environmental Protection, 2011. (14): 14-19.

[2] GUAN Yingzhu, ZHENG Jianhua, and ZHUANG Shijian. Study on the Index System about Ecological Civilization [J]. China Development, 2007, 6 (2): 21- 27.

[3] DU Yu and LIU Junchang. Study on Ecological Civilization Construction Evaluation Index System [J]. Scientific Management Research, 2009, 6 (3): 60-63.

[4] YAN Yezhou and CHENG Jinhua. Analysis of Regional Differences of Ecological Civilization Construction in Hubei Province [J]. Journal of Zhengzhou Institute of Aeronautical Industry Management, 2013, 12 (6): 5-10.

[5] DIAO Shangdong, LIU Yunzhong and CHENG Jinhua. Study on Ecological Civilization Construction Evaluation in Guangzhou City [J]. Decision Reference, 2013 (17): 61-63.

[6] ZHANG Chuanhui and ZHAO Jinling. Chinese Traditional Natural View and Ecological Civilization Education [J]. Journal of Social Science of Jiamusi University, 2007, 3 (2): 114-116.

[7] ZHANG Chuanhui and ZHANG Hanwei. On Ecological Civilization Idea under the Perspective of Marxist Natural View [J]. Academic Exchange, 2008, 8 (8): 13-15.

[8] ZHANG Chuanhui and HAN Wei. Research of Xi Jinping Ecological Thought [J]. Journal of Anhui Agricultural Sciences, 2014, 42 (27): 9654-9656. 